\title{
Formación en ciencia y tecnologías de la información geográfica para el ordenamiento y la gestión territorial en honduras
}

\author{
María Cristina Pineda de Carías y \\ Vilma Lorena Ochoa
}

\section{Resumen:}

La formación en el campo de la ciencia y las tecnologías de la información geográfica particularmente en cartografía, geodesia, fotogrametría, infraestructura de datos espaciales hasta llegar al ordenamiento y la gestión del territorio ha sido una de las principales preocupaciones y tareas de la Facultad de Ciencias Espaciales de la Universidad Nacional Autónoma de Honduras. Por medio de un programa de Maestría en Ordenamiento y Gestión del Territorio realizado con la colaboración internacional del Departamento de Geografía de la Universidad de Alcalá, España desde el 2005, juntos hemos venido desarrollando varias promociones que empiezan a incidir positivamente en el conocimiento y manejo de tecnologías tales como los sistemas de información geográfica, percepción remota y sistemas de geoposicionamiento global para su aplicación en los procesos de ordenamiento territorial de Honduras y la región centroamericana. Juntos también trabajamos para el establecimiento de una carrera de licenciatura, con salidas de técnico en sistemas de información geográfica o en catastro. Teniendo como referente el uso de la información geográfica y el ordenamiento territorial en Honduras en este documento analizamos, algunas de las experiencias académicas del desarrollo del Plan de Estudios de la Maestría en Ordenamiento y Gestión del Territorio, las principales líneas de investigación y vinculación seguidas por sus profesores y estudiantes y el campo profesional de sus graduados. Complementamos esta visión con las experiencias de formación general optativa en el campo de las tecnologías de la información geográfica para todas las carreras universitarias de grado y, el estado de la propuesta de carrera de Ciencia y Tecnologías de la Información Geográfica en el grado de licenciatura. Señalamos el importante papel de la cooperación académica, científica y técnica 
internacional, regional y nacional para el desarrollo de este tipo de programas de formación.

Palabras clave: Ciencia y Tecnologías de la Información Geográfica; Ordenamiento Territorial; Formación Universitaria.

\section{Abstract:}

Training in the field of science and geographic information technologies particularly in cartography, geodesy, photogrammetry, spatial data infrastructure up to the organization and management of the territory has been one of the main concerns and tasks of the Space Science Faculty of the National Autonomous University of Honduras. Through a master's program in Planning and Land Management realized with international collaboration of the Department of Geography, University of Alcalá, Spain since 2005, we have been developing together various promotions that are beginning to have a positive impact on knowledge and management of technologies such as geographic information systems, remote sensing and global positional systems for application in land management processes of Honduras and Central America. Together we also work for the establishment of an undergraduate career, with technical outputs in GIS and cadastre. Taking as reference the use of geographical information and land management in Honduras in this paper we analyze some of the academic experiences of curriculum development of the Master of Planning and Land Management, the main lines of research and outreach followed by teachers and students and the professional field of their graduates. We complement this vision with the general education elective training experiences in the field of geographic information technologies to all university undergraduate careers and, the status of the proposed Science and Geographic Information Technologies undergraduate career. We pointed out the important role of international, regional and national academic, scientific and technical cooperation for the development of this type of training Programs.

Keywords: Science and Geographic Information Technologies; Planning and Land Management; university education.

María Cristina Pineda de Carías (mcpinedacarias@gmail.com); Vilma Lorena Ochoa (vl_ochoa@yahoo.com.mx). Facultad de Ciencias Espaciales, Universidad Nacional Autónoma de Honduras. 


\section{ANTECEDENTES: PRIMERAS INICIATIVAS}

La formación en el campo de la ciencia y las tecnologías de la información geográfica particularmente en cartografía, geodesia, fotogrametría, infraestructura de datos espaciales hasta llegar al ordenamiento y la gestión del territorio ha sido una de las principales preocupaciones y tareas de la Facultad de Ciencias Espaciales de la Universidad Nacional Autónoma de Honduras.

En el año 2005, el Consejo de Educación Superior de la Universidad Nacional Autónoma de Honduras aprobó la creación y funcionamiento de un programa de Maestría en Ordenamiento y Gestión del Territorio (Labortorio Percepción Remota - OACS/UNAH, 2005). El Plan de Estudios de esta Carrera fue elaborado por profesores de la Universidad en colaboración con profesionales de la Unidad Coordinadora de Proyectos de la Secretaría de Gobernación y Justicia de Honduras, dependencia en la que estaba en ese entonces adscrita la Dirección de Ordenamiento Territorial. Participaron también en este equipo, docentes del Departamento de Geografía de la Universidad de Alcalá, España, con quienes desde esas fechas se ha venido trabajando de manera conjunta en la formación de varias promociones que empiezan a incidir positivamente en los procesos de ordenamiento territorial de Honduras y la región centroamericana.

En este artículo se describe como el desarrollo del programa de Maestría en Ordenamiento y Gestión del Territorio se ha convertido en un proyecto de cooperación para el desarrollo, con profesionales que se desempeñan conforme al perfil profesional ocupacional propuesto. Que particularmente en su desempeño en la Universidad Nacional Autónoma de Honduras, algunos de estos profesionales están contribuyendo de manera muy significativa en los procesos de formación de estudiantes de todas las carreras universitarias, pero también innovando el desarrollo curricular con la elaboración de una propuesta de formación en Licenciatura en Ciencia y Tecnologías de la Información Geográfica con salidas de Técnico Universitario en Sistemas de Información Geográfica con énfasis en Catastro. Se destaca la importancia de la cooperación internacional para el desarrollo de este nuevo campo en Honduras.

\section{MOGT: PROYECTO DE COOPERACIÓN PARA EL DESARROLLO}

Objetivos del Programa de Maestría en Ordenamiento y Gestión del Terri- 
torio (MOGT) han sido, de una parte, contribuir a formar personal calificado de alto nivel, comprometido socialmente para desempeñarse con eficacia y eficiencia en la dirección de los procesos de ordenamiento territorial integrado iniciados en Honduras. De otra parte, con el desarrollo de esta carrera se ha buscado fortalecer la infraestructura básica para el funcionamiento de un Laboratorio de Tecnologías de la Información Geográfica, como un modelo de centro especializado para desarrollar actividades académicas y científicas para beneficio de estudiantes, docentes, investigadores del país y la región centroamericana. De esta manera, condiciones fueron creadas para el conocimiento y manejo de tecnologías tales como los sistemas de información geográfica, percepción remota y sistemas de geoposicionamiento global; el laboratorio ha sido dotado con computadoras, programas informáticos para el uso y manejo de datos geoespaciales, material bibliográfico en diferentes formatos, así como de datos, imágenes y mapas regionales, nacionales, departamentales y municipales de diferentes escalas.

El Plan de Estudios de la MOGT comprende diecinueve asignaturas distribuidas en seis ciclos. En la Tabla 1 se presenta el detalle de los ciclos y las asignaturas. Los primeros cinco ciclos de este Programa de Maestría están dedicados a: Geografía y Fundamentos de Ordenamiento Territorial, Geodesia y Cartografía, Sistemas de Información Geográfica, Percepción Remota, Ordenamiento Territorial. El último ciclo, comprende dos modalidades, de una parte incluye una Práctica Profesional aplicada al Ordenamiento Territorial. De otra parte comprende un Proyecto de Investigación, que posteriormente se convierte en la tesis que presentan los estudiantes como requisito de graduación. Esta última característica hace que este programa, sea un híbrido, entre un programa maestría académico y un programa de maestría profesionalizante de posgrado, como lo contemplan las Normas Académicas de Educación Superior de Honduras (Consejo de Educación Superior, 2004) y la común aceptación en el ámbito regional centroamericano. 
TABLA 1. detalle de asignaturas por ciclo de la maestría en ordenamiento y gestión del territorio de la unah.

\begin{tabular}{|c|c|c|c|}
\hline No. & CODIGO & CICLO / ASIGNATURA & $\begin{array}{l}\text { UNIDADES } \\
\text { VALORATIVAS }\end{array}$ \\
\hline & & CICLO 1: GEOGHAFIA Y FUMDAMENTOS DEORDENAMENTOTEFGTOPUAL & \\
\hline 1 & CTE-501 & Geografia y Ordenación del Territorio & 1 \\
\hline \multirow[t]{2}{*}{2} & CTE-502 & Fundamentos de Ordenamiento Territorial & 1 \\
\hline & & CICLO 2: GEOCESIA Y CARTOGRAFIA & \\
\hline 3 & CTE-511 & Fundamentos de Geodesia y Cartografla & 2 \\
\hline 4 & CTE-512 & Fotogrametria y Sistemas de Geoposicionamiento Global & 2 \\
\hline 5 & CTE- 513 & Mapas: Diseño, Composición, Trazado e Impresión & 3 \\
\hline \multirow[t]{2}{*}{6} & CTE-514 & Atlas Electrónicos y Publicación de Mapas en la Web & 3 \\
\hline & & CICLO 3: SISTEMAS DE INFCRMACIONGEOGRAFKCA & \\
\hline 7 & CTE-521 & Fundamentos de Sistemas de Información Geográfica & 2 \\
\hline 8 & CTE-522 & Sistema de Información Geográfidpinster & 2 \\
\hline 9 & CTE-523 & Sistema de Información Geográfickector & 2 \\
\hline \multirow[t]{2}{*}{10} & CTE-524 & $\begin{array}{l}\text { Programaciónplicada a entorno de Sistemas de Información } \\
\text { Geográfica }\end{array}$ & 3 \\
\hline & & CICLO 4: PERCEPCION REMOTA & \\
\hline 11 & CTE-531 & Principios Físicos de Percepción Remota & 2 \\
\hline 12 & CTE-532 & Plataformas, Sensores y Teledetección Hiperespectral & 2 \\
\hline 13 & CTE-533 & Interpretación Visual de Imágenes & 2 \\
\hline \multirow[t]{2}{*}{14} & CTE-534 & Procesamiento e Interpretación Digital de Imágenes & 3 \\
\hline & & CICLO 5: ORDENAMIENIOTERRITOFIAL & \\
\hline 15 & CTE-541 & Administración del Territeraplicaciones & 3 \\
\hline 16 & CTE-542 & Planificación Territoriaplicaciones & 3 \\
\hline \multirow[t]{2}{*}{17} & CTE-543 & Gestión TerritorialAplicaciones & 3 \\
\hline & & CICLL 6: Práctica ProfesionAl. & \\
\hline 18 & CTE 600 & $\begin{array}{l}\text { PrácticaProfesional aplicada al } \\
\text { Ordenamiento Territorial }\end{array}$ & 4 \\
\hline \multirow[t]{2}{*}{19} & CTE-700 & $\begin{array}{l}\text { Proyecto de Investigación } \\
\text { (Tesis de Maestría) }\end{array}$ & 8 \\
\hline & & TOTAL & 51 \\
\hline
\end{tabular}

FUENTE: Plan de Estudios de la MOGT. Elaboración propia.

Desde el año 2005 hasta el 2013 se han desarrollado tres promociones de la MOGT, llamadas respectivamente: MOGT1, MOGT2 y MOGT3. El Programa de la MOGT es codirigido por profesores del Departamento de Ciencia y Tecnología de la Información Geográfica (DCTIG) de la Facultad de Ciencias Espaciales (FACES), y por Profesores del Departamento de Geografía de la Universidad de Alcalá, España. Derivado de esta cooperación con la Universidad de Alcalá, se han incorporado al cuerpo docente, profesores de otras universidades de España, de Argentina, Chile, Costa Rica, México, Nicaragua, Puerto Rico, Venezuela, por lo que la MOGT como programa de posgrado, se ha convertido en un proyecto de cooperación para el desarrollo. En esta condición, forma parte del Programa de Cooperación con Centroamérica de la Universidad de Alcalá. 


\section{LÍNEAS DE INVESTIGACIÓN Y PERFIL OCUPACIONAL DE GRADUADOS}

Utilizando los conocimientos, metodologías y técnicas de investigación aprendidas a lo largo de los diferentes ciclos de estudio de la MOGT, los estudiantes, bajo la dirección de un profesor tutor resuelven un problema específico propio de la Ciencia y las Tecnologías de la Información Geográfica aplicadas al Ordenamiento Territorial. A partir de una investigación bibliográfica realizada en los archivos y documentos de la Coordinación Académica de la MOGT, con este trabajo hemos podido identificar las principales tendencias de investigación de los graduados; y como el perfil profesional contenido en el Plan de Estudios se corresponde con el campo de trabajo de los graduados.

\subsection{Líneas de Investigación}

Dentro del marco del Plan de Estudios y las políticas de investigación de la MOGT, la Coordinación Académica de la carrera ha orientado los temas de tesis de los estudiantes dentro de líneas de investigación claramente definidas. Nuestro estudio de la clasificación de los temas de tesis por líneas de investigación hasta 2013, ha dado los siguientes resultados:

a) Recursos Naturales. Incluye la gestión de los recursos naturales, relacionada con las áreas protegidas, producción y conservación de los recursos hídricos y bosques: $35 \%$.

b) Zonificación y uso del suelo. Incluye Ordenamiento territorial orientado a la zonificación del territorio, el uso del suelo (actual y potencial): $26 \%$.

c) Zonificación Urbana. Incluye zonificación urbana, centros históricos y sitios arqueológicos: $13 \%$.

d) Cartografía. Incluye Cartografía y gestión de riesgos, orientada a incendios, inundaciones, deslizamientos y otros fenómenos naturales o antrópicos: $13 \%$.

e) Geodesia. Incluye Redes Geodésicas: 9\%. 
f) Regionalización. Incluye regionalización nacional, esquemas de ordenamiento territorial: $4 \%$.

FIGURA 1. Líneas de Investigación estudiantes MOGT.

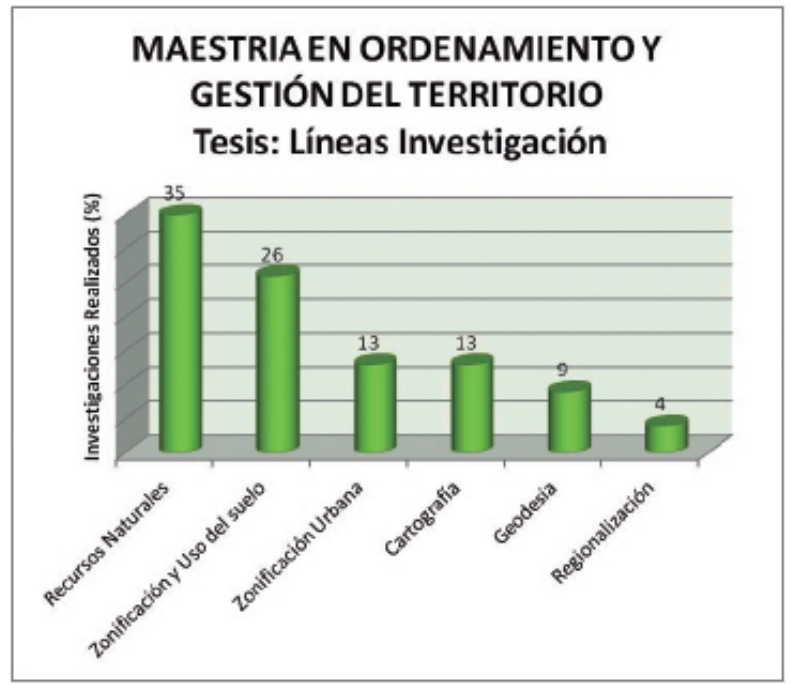

Fuente: Coordinación Académica MOGT. Elaboración propia.

Estos resultados revelan la dominancia de las investigaciones sobre la gestión de los recursos naturales y el ordenamiento territorial orientado a la zonificación y el uso del suelo.

\subsection{Perfil ocupacional de los graduados}

De acuerdo con el Plan de Estudios vigente, el graduado de la Maestría en Ordenamiento y Gestión del Territorio es un profesional con una formación especializada en Ciencia y Tecnología Espacial Básica, con conocimientos, que interrelacionan la teoría con la práctica y la formación académica con la realidad social en Geografía, Geodesia, Cartografía, Sistemas de Información Geográfica, Percepción Remota y Ordenación del Territorio, que sabe aplicarlos de manera autocrítica y propositiva a situaciones administrativas, de gestión y ordenamiento del territorio; que posee además una formación práctica para el manejo y uso de equipos, programas y paquetes de computación para la adquisición, procesamiento, análisis y gestión de datos espaciales, para la elaboración y el desarrollo de planes maestros, proyectos especializados, mapas básicos, catastrales, temáticos y de zonificación en escalas local, municipal, regional y nacional para el ordenamiento integral del 
territorio.

El estudio que hemos realizado para comparar el perfil ocupacional y las oportunidades profesionales de los graduados de la MOGT con los puestos ocupados por los graduados de las tres primeras promociones hasta 2013 , nos ha revelado los resultados que presentamos en la Tabla 2.

\section{TABLA 2. PERFIL PROFESIONAL OCUPACIONAL Y DE DESEMPEÑO GRADUADOS DE LA MOGT POR PROMOCIONES 2013.}

\begin{tabular}{|c|c|c|}
\hline PROMOCION & $\begin{array}{l}\text { PERFIL PROFESIONAL Y OPORTUNIDADES } \\
\text { PROFESIONALES }\end{array}$ & $\begin{array}{l}\text { OCUPACIONES Y DESEMPENO DE } \\
\text { GRADUADOS DE LA MOGT }\end{array}$ \\
\hline \multirow[t]{4}{*}{ MOGT1 } & $\begin{array}{l}\text { Gestor de proyectos en los que se requiera introducir } \\
\text { innovaciones a la forma de plantear soluciones a } \\
\text { problemas, y con un planteamiento moderno y } \\
\text { competitivo. }\end{array}$ & $\begin{array}{l}\text { GIZ Programa RED para Centroamérica y } \\
\text { República dominicana. }\end{array}$ \\
\hline & $\begin{array}{l}\text { Consultor del Sector Privado. Especialista en } \\
\text { procesamiento, interpretación y análisis de datos } \\
\text { gecespaciales. }\end{array}$ & INGTELSIG \\
\hline & Profesor Universitario. Investigador. & DCTIG, FACESIUNAH \\
\hline & Gerencia Administración Municipal. & Gerencia de Catastro AMDC \\
\hline \multirow[t]{7}{*}{ MOGT2 } & Profesor Universitario. Investigador & $\begin{array}{l}\text { Centro de Sistema de Información } \\
\text { Geográfica de Facultad Ciencias Sociales, } \\
\text { UNAN, Nicaragua }\end{array}$ \\
\hline & $\begin{array}{l}\text { Administrador de Proyectos. Especialista en } \\
\text { procesamiento y análisis de datos geoespaciales. }\end{array}$ & $\begin{array}{l}\text { Comisión Permanente de Contingencias } \\
\text { (COPECO) }\end{array}$ \\
\hline & Profesor Universitario. Investigador. & DCTIG, FACESUNAH \\
\hline & Consultor de Ciencia y Tecnologia & $\begin{array}{l}\text { Departamento Edificio Públicos, Secretaria } \\
\text { Obras Públicas, Transporte y Vivienda }\end{array}$ \\
\hline & $\begin{array}{l}\text { Especialista en procesamiento, interpretación y } \\
\text { análisis de datos geoespaciales. }\end{array}$ & $\begin{array}{l}\text { Comisión Especial de Demarcación, } \\
\text { Secretaria Relaciones Exteriores }\end{array}$ \\
\hline & Profesor Universitario. Investigador. & $\begin{array}{l}\text { Laboratorio SIG, Escuela Nacional de } \\
\text { Ciencias Forestales (ESNACIFOR) }\end{array}$ \\
\hline & Profesor Universitario. Investigador. & Departamento Biologia, UNAH \\
\hline \multirow[t]{4}{*}{ MOGT3 } & Profesor Universitario. Investigador. & $\begin{array}{l}\text { Departamento Arqueoastronomia } \\
\text { Astronomia Cultural, FACESUNAH }\end{array}$ \\
\hline & $\begin{array}{l}\text { Consultor del Sector Privador. Especialista en } \\
\text { procesamiento, interpretación y análisis de datos } \\
\text { gecespaciales. }\end{array}$ & INYPHSA \\
\hline & Profesor Universitario. Investigador. & DCTIG, FACESIUNAH \\
\hline & Director General en la Administración Pública & $\begin{array}{l}\text { Dirección General de Catastro y Geografia, } \\
\text { Instituto de la Propiedad. }\end{array}$ \\
\hline
\end{tabular}

FUENTE: Archivos Coordinación MOGT (Coordinación Académica MOGT, 2005 - 2013). Elaboración propia.

Estos resultados revelan que todos los graduados de la MOGT se desempeñan dentro de su campo profesional, ocupando puestos acordes a su formación en la administración pública y municipal, empresa privada, en organismos y empresas nacionales e internacionales, y en la docencia universitaria. 
En la docencia universitaria, los graduados de la MOGT se encuentran realizando investigación y ejerciendo la docencia, a nivel de grado y posgrado. Los campos en que se desempeñan son el Ordenamiento Territorial, desarrollo de las TIG y sus aplicaciones, gestión de riesgos, patrimonio arqueológico, monitoreo de desastres naturales y antropogénicos, aplicaciones forestales, manejo de recursos naturales y áreas silvestres, y ecología de paisaje.

En la administración pública y municipal, los graduados laboran principalmente en los temas de catastro municipal y nacional, la demarcación de las fronteras, y con el análisis de datos geoespaciales en proyectos de gestión de riesgos.

En la empresa privada los graduados de la MOGT, han sido responsables de la coordinación o participación en procesos de ordenamiento territorial, sea a nivel de planificación o a través de los diagnósticos sectoriales mediante la aplicación de las TIG.

Finalmente, a nivel de organismos internacionales, participan como especialista en el procesamiento, interpretación y análisis de datos geoespaciales, en procesos de la regionalización y zonificación, dirigiendo procesos de ordenamiento y fortalecimiento de capacidades y toma de decisiones del sector forestal a nivel centroamericano.

\section{DCTIG: FORMACIÓN GENERAL}

En la Facultad de Ciencias Espaciales de la Universidad Nacional Autónoma de Honduras, se ha llegado a conformar un núcleo de docentes graduados de la Maestría en Ordenamiento y Gestión del Territorio, que ha permitido organizar y poner en funcionamiento el Departamento de Ciencia y Tecnologías de Información Geográfica. Las actividades académicas de estos profesores incluyeron la docencia, la investigación y la vinculación de la universidad con la sociedad.

Para contribuir a la formación general e integral de los estudiantes universitarios de todas las carreras de la UNAH, el Departamento de Ciencia y Tecnologías de la Información Geográfica desde el año 2009 propuso la oferta de tres asignaturas optativas: 1) CTE-111 Introducción a la Percepción Remota; 2) CTE-112 Introducción a los Sistemas de Información Geográfica; 3) CTE-113 Introducción a los Sistemas de Geoposicionamiento Global. La aceptación de los estudiantes 
de estas asignaturas ha sido creciente. En la Figura 1 se presenta un gráfico que muestra la matrícula desde el año de su creación, hasta el segundo período académico de 2013. Estas asignaturas tienen 3 unidades valorativas, y se desarrollan de forma presencial apoyada en una plataforma virtual a la que los estudiantes pueden acceder por Internet, y contempla clases teóricas y actividades prácticas realizadas en el Laboratorio de Tecnologías de la Información Geográfica de grado.

FIGURA 2. Matrícula asignaturas generales CTE111, CTE112 y CTE113.

Período: 2009 a Julio-2013.

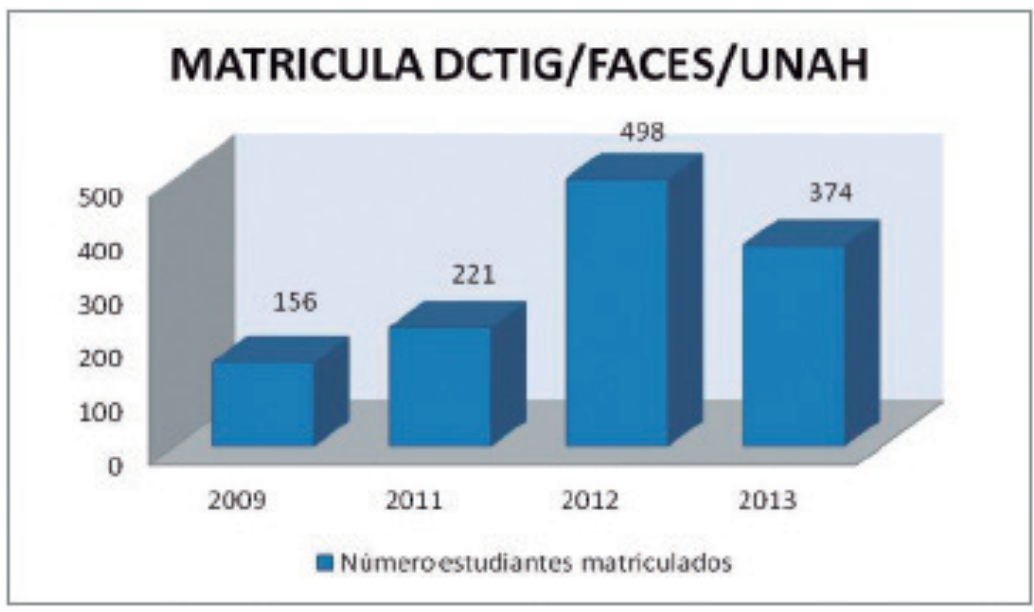

Fuente: Estadísticas matrícula DCTIG (Departamento Ciencia y Tecnologías de la Información Geográfica, 2009 - 2013). Elaboración propia.

\section{UNA CARRERA DE GRADO EN CTIG NUEVA}

La Universidad Nacional Autónoma de Honduras es una institución autónoma del Estado que goza de la exclusividad de organizar, dirigir y desarrollar la educación superior y profesional del país. En sus procesos de reforma reciente, la UNAH ha aprobado un Modelo Educativo (Rectoría, Vicerrectoría Académica, Comité Técnico de Apoyo al Desarrollo Curricular, 2009) que responde a ideales políticos que simbolizan los intereses de Honduras como nación: innovación, creatividad, respuesta a la demanda de cambio, necesidad de transformación hacia la modernización y democratización. El modelo se conceptúa como: 
- La forma totalizadora en que una comunidad educativa histórica y culturalmente situada, siente, piensa, organiza su quehacer haciendo realidad el hecho educativo.

- Es el instrumento de trabajo, que permite una visión sistemática y coherente de los procesos educativos que surgen en la comunidad.

- Permite orienta la práctica, entender la acción, y reorientarla en una dinámica permanente de teoría-práctica-teoría.

Principios básicos que sustentan el Modelo Educativo de la UNAH son: la calidad, pertinencia, equidad, interdisciplinariedad, internacionalización. Su perspectiva pedagógica hA tomado como base: la Teoría Constructivista, la Teoría Crítico - Reflexiva y la Teoría Humanista.

Con este marco institucional, y orientados por el Modelo Educativo, la Facultad de Ciencias Espaciales ha priorizado el proceso de desarrollo curricular en los campos que le son propios, y dentro del que se ubica el de la Ciencia y las Tecnologías de la Información Geográfica. El proceso de desarrollo curricular es un proceso de construcción dinámico y permanente en el que se conjugan las diversas relaciones entre los actores universitarios, metodologías, estrategias, recursos, medio y contenidos disciplinarios orientados a la formación de profesionales capaces de contribuir a transformar la realidad social hondureña, a través de sus aportes a la superación de los problemas prioritarios que el país enfrenta en la actualidad y que enfrentará en el futuro.

Siguiendo el Modelo Educativo de la UNAH, y el proceso de desarrollo curricular planteado por la FACES, el Departamento de Ciencia y Tecnologías de la Información Geográfica, ha planteado una propuesta de creación y puesta en funcionamiento de una Licenciatura en Ciencia y Tecnologías de la Información Geográfica, con una opción de salida temprana de Técnico Universitario en Sistemas de Información Geográfica con énfasis en Catastro (Departamento Ciencia y Tecnologías de la Información Geográfica, 2013). Este proyecto ha sido el resultado de una investigación educativa. En la Figura 2 se presenta un diagrama del proceso de desarrollo curricular. 
FIGURA 3. Modelo Educativo UNAH. Proceso desarrollo curricular LCTIG.

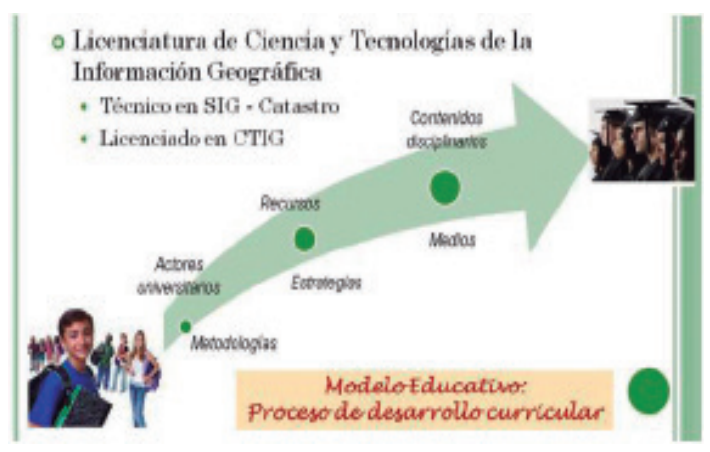

El perfil del profesional de Ciencia y Tecnologías de la Información Geográfica incluye, entre otras, las siguientes funciones, tareas y/o responsabilidades:

- Generar, editar y analizar datos geoespaciales.

- Diseño y administración de bases de datos espaciales.

- Diseño, desarrollo y administración de sistemas de información geográfica.

- Análisis e interpretación de productos derivados de sensores remotos para la generación de geoinformación.

- Generación y manejo de modelos digitales de terreno.

- Planificación y ejecución de trabajos de campo para la adquisición y actualización de datos geoespaciales.

- Levantamientos topográficos mediante tecnologías de posicionamiento global.

- Generación de productos cartográficos.

- Generación de aplicaciones Geoinformáticas. 
- Diseño de proyecto de desarrollo para solución de problemas de tipo geográfico.

- Apoyo técnico profesional en la elaboración de sistemas automatizados para planes de ordenamiento territorial, evaluación y gestión de riesgos naturales, manejo de áreas protegidas y recursos naturales.

El proyecto de creación de la carrera de Licenciatura en Ciencia y Tecnologías de la Información Geográfica está en proceso de aprobación de las instancias correspondientes en la Universidad Nacional Autónoma de Honduras.

\section{CONCLUSIONES}

- El campo de la ciencia y las tecnologías de la información geográfica se ha establecido en la Universidad Nacional Autónoma de Honduras a nivel de grado y posgrado.

- A nivel de grado, la educación general incrementa su demanda para servicio de todas las carreras universitarias. Hay demanda de una carrera en el campo de la Ciencia y las Tecnologías de la Información Geográfica con salidas tempranas a nivel de técnico universitario.

- En la Maestría en Ordenamiento y Gestión del Territorio, sus egresados empiezan a tener impacto en instituciones educativas de nivel superior, gubernamental y municipal, y en empresas privadas, a nivel nacional e internacional.

- Se reconoce el importante papel de la cooperación académica, científica y técnica nacional e internacional para el desarrollo de programas de formación universitaria de grado y posgrado

\section{REFERENCIAS BIBLIOGRÁFICAS}

- Consejo de Educación Superior. (2004). Normas Académicas del Nivel de Educación Superior. Tegucigalpa: Dirección Educación Superior, UNAH.

- Coordinación Académica MOGT. (1 - 31 de Enero a Diciembre de 2005 - 2013). Informes, Documentos, Memorias y Correspondencia. Documentos Maestría en Ordenamiento y Gestión del Territorio. Tegucigalpa, Francisco Morazán, Honduras: Maestría en Ordenamiento y Gestión del Territorio. 
- Departamento Ciencia y Tecnologías de la Información Geográfica. (1 - 31 de Enero - Diciembre de 2009 - 2013). Documentos, Informes, Memorias, Correspondencia. Documento. Tegucigalpa, Francisco Morazán, Honduras: Departamento Ciencia y Tecnologías de la Información Geográfica, FACES/UNAH.

- Departamento Ciencia y Tecnologías de la Información Geográfica. (2013). Propuesta de Plan de Estudios de la Carrera de Ciencia y Tecnologías de la Información Geográfica en el Grado de Licenciatura. Tegucigalpa: Departamento Ciencia y Tecnologías de la Información Geográfica, FACES/UNAH.

- Labortorio Percepción Remota - OACS/UNAH. (2005). Plan de Estudios de la Carrera de Ordenamiento y Gestión del Territorio en el Grado de Maestría. Tegucigalpa: OACS/UNAH, Tegucigalpa, Honduras.

- Rectoría, Vicerrectoría Académica, Comité Técnico de Apoyo al Desarrollo Curricular. (2009). El Modelo Educativo de la UNAH. Tegucigalpa: Universidad Nacional Autónoma de Honduras. 